\title{
Differential regulation of septation
}

DOI:

10.1038/nrmicro1733
In filamentous fungi septation occurs during both vegetative growth and conidiation. New results recently published in Eukaryotic Cell now indicate that in Neurospora crassa these two septation pathways are differentially regulated.

Previous work in N. crassa had shown that the Rho-type GTPase RHO-4 persistently localizes to vegetative septa and is required for septation. Carolyn Rasmussen and Louise Glass were interested in further probing the involvement of RHO-4 in N. crassa septation, and to do so they generated a green fluorescent protein (GFP)-labelled version of the protein and tracked its localization during $N$. crassa growth and development.

Initial analysis revealed that during vegetative septum formation, RHO-4 localized to the plasma membrane, forming a wide ring at the incipient septum site that constricted during septum formation. RHO-4 persisted as a ring around the septal pore after septum formation was complete. During asexual spore formation (conidiation), two complete septa are formed: primary septa, which form after the development of aerial hyphae, and secondary septa, which separate the conidia (this is known as the double-doublet stage). The localization of RHO-4 during conidiation was also observed and it was found that RHO-4 transiently localized to the primary septa. However, this localization changed during conidiation and by the double-doublet stage $\mathrm{RHO}-4$ was located in the cytoplasm.

What was responsible for the differential localization of RHO-4 during conidial septation? Rasmussen and Glass wondered whether a negative regulator was involved and identified the predicted protein NCU06561 as a possible negative regulator based on homology with the Schizosaccharomyces pombe guanine-nucleotide dissociation inhibitor (GDI) RDI-1. Disruption of the NCU06561 open-reading frame led to an increase in both vegetative septation and the plasma membrane localization of RHO-4. Together with the results of immunoprecipitation experiments, which demonstrated a direct interaction between NCU0651 and $\mathrm{RHO}-4$, these data indicate that NCU06561 is a negative regulator of RHO- 4 and sequesters RHO- 4 in the cytoplasm.

Finally, cyclic AMP-dependent protein kinase A (PKA) can affect the interaction between a Rho-type GTPase and its cognate GDI. Rasmussen and Glass therefore looked at RHO-4 localization in $N$. crassa mutants in which the PKA pathway was perturbed and the results were consistent with a scenario in which the CAMP-PKA pathway has a role in regulating both septation pathways, but this role is likely to be different in each pathway. Future work will be directed at further teasing apart the similarities and differences in the two septation pathways.

Sheilagh Molloy

ORIGINAL RESEARCH PAPER Rasmussen, C. G. \& Glass, N. L. Localization of RHO-4 indicates differential regulation of conidial versus vegetative septation in the filamentous fungus Neurospora crassa. Euk. Cell 6, 1097-1107 (2007)

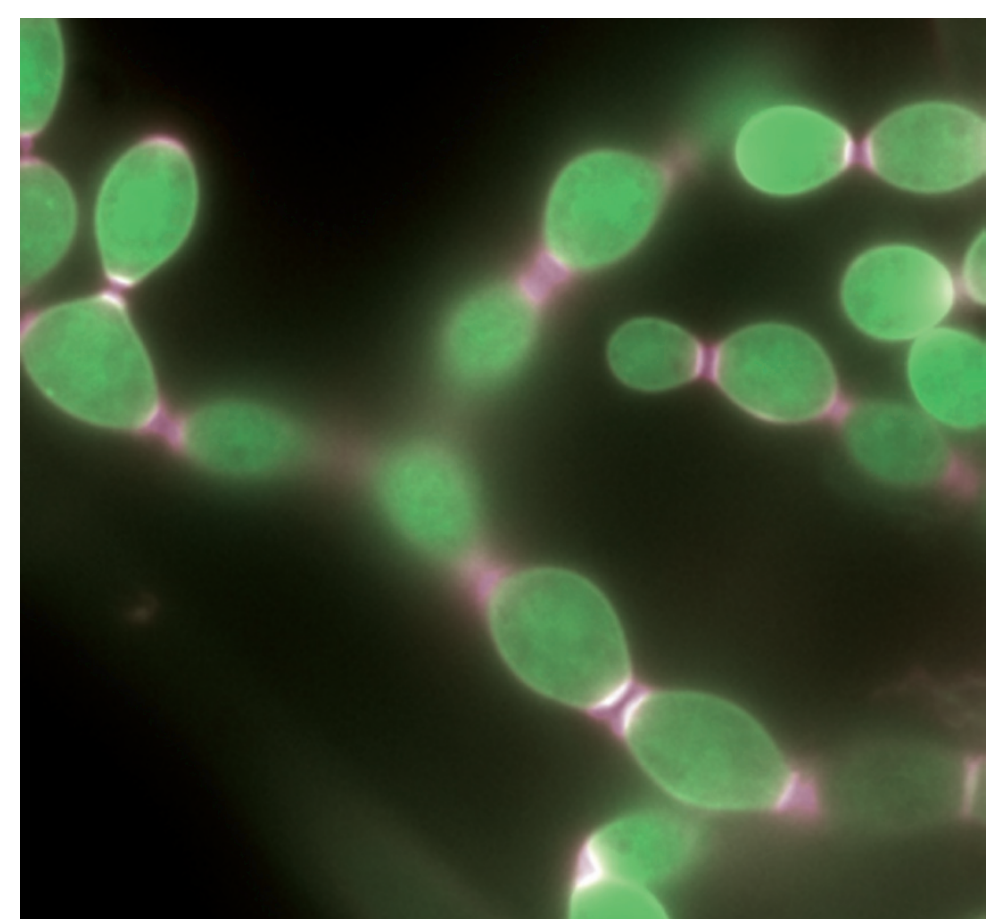

Conidia stained with calcofluor (magenta) and green fluorescent protein-RHO-4 (green). Image kindly provided by Carolyn Rasmussen, University of California, Berkeley, USA. 